\title{
The Exact Evaluation of Basic Two-Center Coulomb Integrals Appearing in Intermolecular Electrostatic Interaction Energy in Molecular Coordinate System
}

\author{
Ebru Çopuroğlu ${ }^{1}$ and Bahtiyar Mamedov ${ }^{2}$ \\ ${ }^{1}$ Tokat Gaziosmanpasa Universitesi \\ ${ }^{2}$ University
}

September 29, 2021

\begin{abstract}
We proposed a general and effective approach for accurate calculating method of the electron-electron, nuclear-electron and nuclear-nuclear Coulomb electrostatic interaction energies. It is well known that electron-electron, nuclear-electron and nuclearnuclear Coulomb electrostatic interaction energies reduced to basic two-center Coulomb integrals. The analytical calculation of electrostatic interaction energies with respect to basic two-center Coulomb integrals over Slater type orbitals (STOs) in molecular coordinate systems allows us the routine evaluation of molecular structures and related properties. In this study we have introduced a new full analytical algorithm for calculation of the basic two-center Coulomb integrals over STOs by using Guseinov's auxiliary functions especially interactions between electrons. The auxiliary functions has been calculated by using the exact recurrence relations which developed by Guseinov. The new approach is successfully tested on earlier published studies data and can be recommended for evaluation of related problems in atomic and molecular physics.
\end{abstract}

\section{Hosted file}

Two-center Coulomb mkl son.docx available at https://authorea.com/users/438570/articles/ 539743-the-exact-evaluation-of-basic-two-center-coulomb-integrals-appearing-inintermolecular-electrostatic-interaction-energy-in-molecular-coordinate-system 